\title{
Component-resolved diagnosis of pollen allergy based on skin testing with profilin, polcalcin and lipid transfer protein pan-allergens
}

\author{
D. Barber ,F. de la Torre , M. Lombardero, I. Antepara , C. Colas , I. Dávila , A. I. Tabar , C. Vidal . M. Villalba , G. Salcedo and \\ R. Rodriguez \\ Departamento de 11 D, ALK-Abello Madrid, Spain, Medical Marketing, ALK-Abello, Madrid, Spain, Servicio de Alergia, Hospital Basurto, Vizcaya, Spain, ${ }^{4}$ Servicio de \\ Alergia, Hospital Clínico, Zaragoza, Spain, Servicio de Alergia, Hospital Universitario, Salamanca, Spain, Servicio de Alergia, Hospital Virgen del Camino, Pamplona, \\ Spain, Servicio de Alergia, Hospital Conxo, Coruña, Spain, Departamento de Bioquímica y Biología Molecular, Facultad de Ciencias Qurmicas, Universidad \\ Complutense, Madrid, Spain and Unidad de Bioquímica, Departamento de Biotecnologla, ETS Ingenieros Agrónomos, Universidad Politécnica, Madrid, Spain
}

\begin{abstract}
Summary
Background Allergy diagnosis needs to be improved in patients suffering from pollen polysensitization due to the existence of possible confounding factors in this type of patients. Objective To evaluate new diagnostic strategies by comparing skin responses to pan-allergens and conventional allergenic extracts with specific $\operatorname{lgE}(\mathrm{slgE}$ ) to purified allergen molecules. Methods One thousand three hundred and twenty-nine pollen-allergic patients were diagnosed by a combination of an in vitro method with a panel of 13 purified allergens, including major allergens and pan-allergens, using a high-capacity screening technology (ADVIA-Centaur ${ }^{\mathrm{s}}$ ) and skin prick test (SPT) to pan-allergens and conventional extracts. Results There was a high concordance ( $\mathrm{k}$ index) between in vitro (slgE to major allergens) and in vivo (SPT to conventional extracts) methods in patients who were not sensitized to panallergens, but SPT with conventional extracts failed to diagnose patients with sensitization to pan-allergens. In patients who were simultaneously sensitized to polcalcins and profilins, there was a duplication both in the number of sensitizations to major allergens and in the years of disease evolution. There was a statistical association between sensitization to profilins and/or lipid transfer proteins and food allergy (Po0.0001).

Conclusion The novel diagnostic strategy has proven to be a valuable tool in daily clinical practice. Introduction of routine SPT to pan-allergens is a simple and feasible way of improving diagnostic efficacy. Patients sensitized to pan-allergens should be tested by an adequate panel of allergenic molecules in order to identify the allergens that are responsible for the allergic disease.
\end{abstract}

Keywords component-resolved diagnosis, lipid-transfer protein, pan-allergen, polcalcin, profilin

\section{Introduction}

Sensitization to pan-allergens is relatively common in pollen-allergic patients. In a previous study, between $10 \%$ and $50 \%$ of pollen-allergic patients were sensitized to profilin, while up to $10 \%$ of patients were sensitized to polcalcins and lipid transfer protein (LTP) [1]. Patients sensitized to pan-allergens frequently yield multiple positive reactions when extract-based diagnostic techniques are used [2]. In areas where pollinosis is a common disease, patients are frequently exposed to different pol- lens with different sensitizing capacities and most of the times with overlapping pollen seasons. In these cases, it is crucial to identify which pollens are actually responsible for the allergic symptoms in order to establish a correct aetiological treatment. Major allergens are defined as those to which more than $50 \%$ of patients allergic to a particular source are sensitized to [3], but they are usually detected in the vast majority of patients and are markers of primary sensitization [4]. Moreover, most of the specific $\lg \mathrm{E}(\mathrm{s} \lg \mathrm{E})$ to the species is directed to major allergens on a quantitative basis $[5,6]$. Minor allergens are those to 
which $050 \%$ of patients show sensitization. Pan-allergens constitute families of homologous and structurally related proteins from different species (i.e. profilins, lipidtransfer proteins, polcalcins) [7-11] that are responsible for extensive IgE cross-reactivity among a variety of allergenic sources that are not necessarily phylogenetically related. In a recent epidemiological study using single allergens [1], it was demonstrated that some panallergens, such as profilins, behaved as minor grass allergens and thus, in most cases, sensitization to these could be considered as an indicator of a stronger grass allergy sensitization. In the same study, polcalcins did not appear to be linked to any particular pollen sensitization and a third pan-allergen group, fruit lipid-transfer proteins, which had a high prevalence in the study population, was not linked to any specific pollen but caused respiratory symptoms in the study sample. We believe that a routine skin prick test (SPT) with these three pan-allergens could notably improve the correct diagnosis of pollenallergic patients. Moreover, we attempted to find clues on the natural evolution of pollen sensitization and the link between pollinosis and food allergy. This study is an epidemiological survey conducted in Spain, to complement the above-mentioned study [1]. The survey sample consisted of 1329 patients, homogeneously distributed in the study territory, with clinical symptoms compatible with seasonal allergic respiratory disease. SPT to panallergens as well as to conventional allergen extracts were performed on each patient and molecular slgE profiles were obtained. The aim of the study was to propose an improved diagnostic algorithm for pollinosis in a large sample distributed in a diverse pollen territory.

\section{Methods}

\section{Geographical study area}

The area covered by the study is shown in Fig. 1, and represents approximately $50 \%$ of Spain. Most patients were located in the humid Atlantic Ocean area of influence, where allergy to grasses is predominant. This area is climatically representative of an extensive central European region. Other climatic regions such as Mediterranean, dry plateau and subtropical areas were also included. In these areas, the Olive pollen season coincides with the grass and plantain pollen seasons, the Salsola pollen season occurs in late summer, while the Cupressus pollen season occurs from January to February. Parietaria has a broad pollen season from April to August.

\section{Patients}

One thousand three hundred and twenty-nine patients recruited by 57 different clinical groups were studied. About 50 patients were included per province (Fig. 1).
Patients were consecutively selected in a period of 3 months out of the pollen season (September-December). All patients had to have at least a previous 2-year clinical history compatible with pollinosis, meaning, presence of rhinitis, rhino-conjunctivitis or asthma during the pollen season. They should not have received immunotherapy and they should have been living in the province under study for at least the past 5 years. In order to avoid any bias in patient selection, neither specific IgE nor SPTs were performed before patient inclusion. Data were collected by means of a standardized questionnaire. Subjects provided written informed consent and Ethics Committee approvals were obtained.

Patient data were collected in accordance with the clinical group's routine practice. Each patient's serum sample and questionnaire were identified by means of a numeric, barcode label system.

Serum samples were collected from the subjects, identified by the barcode labels, stored at $* 401 \mathrm{C}$ and thawed immediately before use. SPTs were performed in duplicate on the inner forearm by conventional procedures using an ALK-Abello lancet (ALK-Abello S.A., Horsholm, Denmark).

\section{Skin prick test extracts}

Natural profilin, Pho d 2 SPT was prepared by purifying a date palm extract by affinity chromatography with a poly-L-proline-Sepharose. Purity (499\%) was checked by SDS-PAGE, mass spectrometry and amino acid analysis. The concentration of Pho d 2 in the extract was $50 \mathrm{mg} / \mathrm{mL}$. Date palm polcalcin-enriched SPT was obtained from the same extract (showing a total protein concentration of $500 \mathrm{mg} / \mathrm{mL}$ after Lowry) after the removal of profilin. In a previous experiment, positive-SPT responses to complete Palm tree extracts were assigned either to profilin- or to polcalcin-sensitized patients, but not to LTPS, CCDs or Glucanases. Protein identity was assessed by SDS-PAGE. The concentration of polcalcin, measured by an inhibition assay against rChe a 3 , was determined to be $1 \mathrm{mg} / \mathrm{mL}$.

A peach commercial extract from ALK-Abello S.A., adjusted to $30 \mathrm{mg} / \mathrm{mL}$ of Pru p 3, was shown to lack other relevant allergens (such as Pru $p 1$ and Pru p 4).

The rest of the diagnostics were complete pollen commercial extracts from ALK-Abello S.A. at $30 \mathrm{HEP} / \mathrm{mL}$.

\section{Purified allergen panels for specific immunoglobulin $E$}

The following isolated allergens were included: Phleum pratense $n \mathrm{Phl} p 1$ [12] and $n \mathrm{Phl} p 5$ [13]; Artemisia vulgaris nArt v 1 [14]; Olea europaea nole e 1 [15]; Plantago lanceolata nPla I 1 [16]; Parietaria judaica $\mathrm{nPar}$ j 1 [17]; Cupressus sempervirens nCup s 1 [18]; Salsola kali nSal k 1 [19]; and Betula verrucosa nBet v 1 [20]. The following pan-allergens were included: Polcalcin, rChe a 
Table 1. Characteristics of the patient sample

\begin{tabular}{lc}
\hline & $\%$ \\
\hline Age & \\
414 years & 14 \\
$\times 15$ years & 86 \\
Sex & \\
Male & 52.0 \\
Female & 48.0 \\
Symptoms & \\
Rhinitis & 56.0 \\
Asthma" & 1.0 \\
Rhinitis and asthma & 43.0 \\
Food allergy symptoms & \\
Oral allergy syndrome & 15.2 \\
Anaphylactic reaction & 1.4 \\
Urticaria/angio-oedema & 3.6 \\
Pruritus/atopic dermatitis & 0.8 \\
Rhinitis/asthma & 0.8 \\
Gastro-intestinal symptoms & 0.6 \\
Others & 0.08 \\
Unspecific & 1.6 \\
Total & 19.19 \\
\hline
\end{tabular}

According to ARIA definition.

"According to GINA definition.

patients reported food allergy symptoms. $15.2 \%$ reported oral allergy syndrome (OAS), while $8.9 \%$ experienced food allergy symptoms other than OAS.

\section{Sensitization to major allergens}

As expected, grass allergy dominated the sensitization of patients in the study territory. The overall percentages of sensitization to the major grass pollen allergens $\mathbf{P h l} p 1$ and $\mathrm{Phl} \mathrm{p} 5$ were $74.8 \%$ and $48.7 \%$, respectively. Olive sensitization ranked second in prevalence $(29.8 \%)$. The rest of the pollens, as monitored by SIgE to the corresponding major allergens, sensitized a significant, but lower, percentage of patients. Figure 2 shows the geographical distribution of major allergen sensitization. There was a significant Ole e 1 prevalence in regions in the vicinity of olive crop areas but without extensive olive crops. Sal $\mathrm{k} 1 \mathrm{had}$ a significant prevalence in dry regions. Interestingly, the highest prevalence to Par $\mathbf{j} 1$ was detected in certain areas of the Atlantic regions and in Tenerife (Canary Islands) and thus it cannot be considered to be solely a Mediterranean allergen. Pla I 1 ranked second after grass allergens in an extensive area. Finally, Bet $v 1$ prevalence was significant only in the Northwestern region (Galicia) of the study territory.

\section{Sensitization to pan-allergens}

Figure 3 shows the slgE median values and the prevalence of sensitization to the three pan-allergens under study.
The most frequent pan-allergen was profilin (Mal d 4), whose prevalence was $18.8 \%$ in the study area. There was an increased risk of grass pollen allergy in patients sensitized to profilin (OR of 5 associated with $\mathrm{Phl}$ p 5), thus confirming the link between profilin sensitization and grass pollen allergy as described previously [1].

The prevalence of LTPS was significant in all study areas, with an average prevalence of $13 \%$ in the pollenallergic population, despite the fact that plant food allergy was not an inclusion criterion. Prevalence was almost twice as high in the paediatric group (O14 years old), in comparison with the rest of the sample ( $20.8 \%$ vs. $12.2 \%$, $P=0.0035)$. There was no statistical association with any major pollen allergen or with the other LTPs included in the study (Par j 1), in agreement with their low amino acid sequence identity [11].

Interestingly, two areas with extreme differences were identified. In the unique area, where a significant prevalence of sigE to Bet $v 1$ was detected, Pru $p 3$ prevalence was close to $2 \%$. At the other end of the scale, there were areas where Pru p 3 prevalence was in the range of $40 \%$. In these areas, with extensive crops of orchard trees, a significant percentage $(10 \%)$ of the study patients were monosensitized to Pru $\mathrm{p} \mathrm{3}$, suggesting that as patients were selected based on the presence of respiratory symptoms, the inhalation route could be a significant entry gate to LTP food-associated allergy

The overall prevalence of polcalcin (Che a 3 ) was found to be low $(5.8 \%)$ and there was a statistical association with the English plantain major allergen. The odds ratio of positivity to Pla I 1 in polcalcin-positive patients was 2.8 for a value of slgE to Pla I 1 below $10 \mathrm{kU} / \mathrm{L}$ and 5 for a value of slgE to Pla I 1above $20 \mathrm{~kJ} / \mathrm{L}$. There was a clear and unexpected association between polcalcin sensitivity and increasing sigE levels to Pru $p 3$. In this way, the $O R$ of Pru p 3 positivity in polcalcin-allergic patients increased progressively from 2.1 (cut-off sigE to Pru $p 3$ of $0.35 \mathrm{kU} / \mathrm{L}$ ) to 8.6 (cut-off sigE to Prup 3 of $50 \mathrm{kU} / \mathrm{L}$ ).

We further investigated the association between sensitization to two pan-allergens (profilin and polcalcin) and the degree of polysensitization (as determined by the number of major allergens recognized) and disease progression. As shown in Table 2, pan-allergen sensitization could be a useful marker of disease progression. The progression time of the allergic disease according to the data collected in the questionnaire was higher in patients sensitized to pan-allergens than in patients not sensitized to them (Po0.0001). In order to detect which groups showed statistically significant differences, the StudentNewman-Keuls test was applied. Patients sensitized to both profilin and polcalcin had a higher disease progression time, which was statistically significant in comparison with patients not sensitized to any of them. 


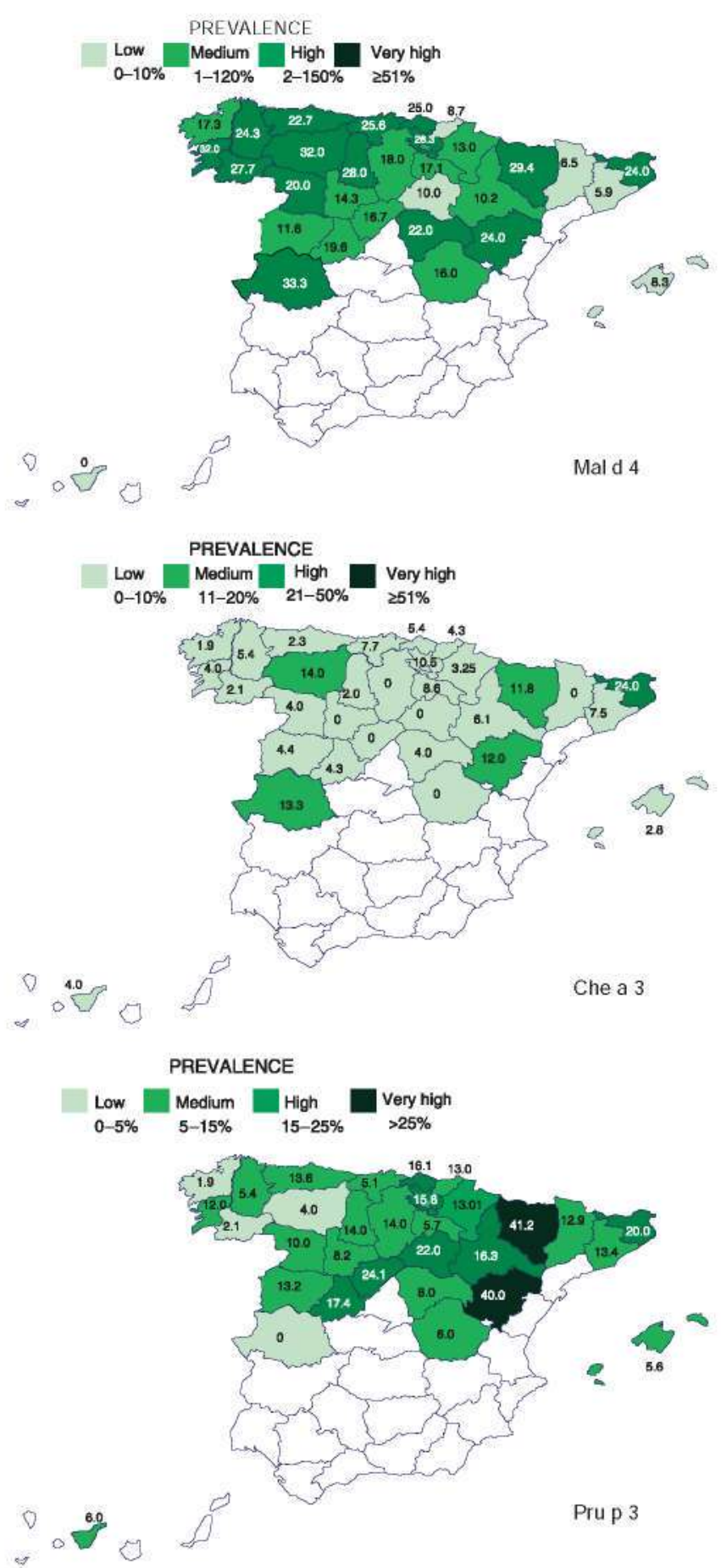

Fig. 3. Prevalence of pan-allergen sigE (profilin, Mal d 4; polcalcin, Che a 3; and LTP, Pru p 3) in the study area. Prevalence categories are defined as indicated, according to a colour scale. Numbers indicate the median slgE value of positive samples.

Analysing the number of sensitizations based on slgE to major allergens and applying the same methodology, the patients sensitized to both the pan-allergens presented a higher number of sensitizations than patients without pan-allergen sensitization.
Pan-allergen sensitization and skin prick test diagnostic performance

Table 3 shows the influence of polcalcin and profilin sensitization on the conventional diagnosis based on an SPT with whole extracts. The analysis was performed by classifying the sample according to pan-allergen positivity. With the exception of Cupressus arizonica pollen sensitization, whenever sensitization to either profilin or polcalcin was present, a lack of specificity of the conventional extract-based SPT was observed. However, SPT to conventional extracts had a high diagnostic value when sensitization to pan-allergens was negative.

Either pure profilin or enriched polcalcin and LTP extracts were tested on SPT. The concordance of these SPT extracts and slgE to the corresponding pan-allergens was evaluated. A high diagnostic value is observed for profilin SPT (positive concordance $82.3 \%$ and negative concordance 90.8\%) and LTP-enriched SPT (positive concordance $65 \%$ and negative concordance $94.3 \%$ ), while polcalcin SPT performance was lower (positive concordance $50 \%$ and negative concordance $90.4 \%$ ).

\section{Food allergy}

The association between food pan-allergen (profilin and LTP) sensitization and food allergy symptoms was evaluated. As the patients were selected on the basis of seasonal allergy symptoms and not food allergy symptoms, this study provides a good opportunity to analyse the risk factors associated with pan-allergen sensitization. A close relationship was observed between sensitization to pan-allergens and food allergy.

Table 4 shows the food allergy symptoms of four groups of patients sensitized to different pan-allergen combinations. From the tabulated data, it is clear that Pru p 3 sensitization is likely to be associated with an increased risk of food allergy both in prevalence and severity. On the other hand, profilin positivity represented only a moderate risk of having mild allergy symptoms. The majority of profilin-positive LTP-negative patients did not show food allergy symptoms.

\section{Discussion}

A correct diagnosis of allergic patients is crucial for a successful specific therapeutic approach. In recent years, a better understanding of immunotherapy mechanisms has led to improved generation of therapeutic allergy vaccines. However, the diagnostic management of polysensitized patients remains largely unchanged. SPT and/or specific IgE to complete extracts, together with a careful patient anamnesis, constitute the basis for diagnosis at present. Pan-allergens are families of cross-reactive molecules present in multiple biological sources that are the 
Table 2. Relationship between sensitization to profilin (Mal d 4) and polcalcin (Che a 3) and polysensitization according to major allergen slgE positivity and years of disease evolution

\begin{tabular}{|c|c|c|c|c|c|c|}
\hline & \multicolumn{3}{|c|}{ Polysensitization (number of positive major allergens by slgE) } & \multicolumn{3}{|c|}{ Years of disease evolution } \\
\hline & $\mathrm{n}$ & Median & $\mathrm{Cl} 95 \%$ & $n$ & Median & $\mathrm{Cl} 95 \%$ \\
\hline PROF (1) POLC (1) & 33 & 5 & $4.04-5.29$ & 28 & 10 & $9.17-15.33$ \\
\hline PROF (1) POLC (*) & 210 & 3 & $2.7-3.06$ & 172 & 6 & $7.26-9.18$ \\
\hline PROF (-) POLC (1) & 44 & 2 & $2.22-3.17$ & 35 & 7 & $7.65-12.29$ \\
\hline \multirow[t]{2}{*}{ PROF (•) POLC (•) } & 1001 & 2 & $1.87-2.03$ & 818 & 5 & $6.95-7.79$ \\
\hline & \multicolumn{3}{|c|}{$\mathrm{P}$ (Kruskal-Wallis) 00.0001} & \multicolumn{3}{|c|}{ P (Kruskal-Wallis) 00.0001} \\
\hline
\end{tabular}

Cl 95\%, confidence interval 95\%; PROF, profilin; POLC, polcalcin.

Table 3. Level of concordance ( $k$ index) between SPT to extracts and slgE to major allergens, according to sensitization to profilin (Mal d 4), polcalcin (Che a 3 ) or negative to both pan-allergens

\begin{tabular}{lllc}
\hline & $k$ (P-value) & & \\
\cline { 2 - 4 } & Profilin (1) & Polcalcin (1) & Profilin (* )/polcalcin (*) \\
\hline Artemisia & 0.1663 & 0.0069 & 0.5199 \\
& $(00.0028)$ & (NS) & $(00.0001)$ \\
Betula & 0.1104 & 0.0270 & 0.5988 \\
& (NS) & (NS) & $(00.0001)$ \\
Cupressus & 0.5584 & 0.6086 & 0.5994 \\
& $(00.0001)$ & $(00.0001)$ & $(00.0001)$ \\
Olea & 0.2719 & 0.0697 & 0.5289 \\
& $(00.0001)$ & $(\mathrm{NS})$ & $(00.0001)$ \\
Parietaria & 0.1668 & 0.0656 & 0.5512 \\
& $(00.036)$ & $(\mathrm{NS})$ & $(00.0001)$ \\
Grass & 0.0713 & 0.2855 & 0.6791 \\
& (NS) & $(00.011)$ & $(00.0001)$ \\
Plantago & 0.0235 & 0.0709 & 0.2583 \\
& (NS) & (NS) & $(00.0001)$ \\
Salsola & 0.1662 & 0.0442 & 0.5678 \\
& $(00.0004)$ & (NS) & $(00.0001)$ \\
\hline
\end{tabular}

NS, not significant; SPT, skin prick test; slgE, specific lgE.

main cause of diagnostic failure when using complete extracts. The introduction of component-resolved diagnosis is providing new diagnostic tools that will hopefully lead to the identification of an accurate patient sensitization profile [26]. However, from a pharmacoeconomic point of view, the mass testing of all allergic patients with these new tools is too costly. Thus, one of the principal objectives of this study was to evaluate a new diagnostic strategy where the introduction of new SPTs specific for three pan-allergen groups, together with an appropriate and complete panel of allergenic molecules, could lead to an improved diagnostic algorithm. As a secondary objective, we aimed to investigate the link between pollen and food allergies.

In this study, we observed that patients who reside in areas where grass pollen dominates are usually monosen-
Table 4. Pan-allergen sensitization and food allergy

\begin{tabular}{|c|c|c|c|c|c|c|c|c|c|}
\hline \multirow[b]{3}{*}{ Oral allergy } & \multicolumn{8}{|c|}{ Profilins (Mal d 4) and LTPs (Prup 3) } & \multirow{3}{*}{$\begin{array}{l}\text { - Total } \\
\text { (\%) }\end{array}$} \\
\hline & \multicolumn{2}{|c|}{$\begin{array}{l}\text { PROF1 } \\
\text { LTP1 }\end{array}$} & \multicolumn{2}{|c|}{$\begin{array}{l}\text { PROF } 1 \\
\text { LTP. }\end{array}$} & \multicolumn{2}{|c|}{$\begin{array}{l}\text { PROF. } \\
\text { LTP1 }\end{array}$} & \multicolumn{2}{|c|}{$\begin{array}{l}\text { PROF. } \\
\text { LTP. }\end{array}$} & \\
\hline & $N$ & $\%$ & $\mathrm{~N}$ & $\%$ & $\mathrm{~N}$ & $\%$ & $\mathrm{~N}$ & $\%$ & \\
\hline OAS & 13 & 30.23 & 50 & 25.00 & 26 & 20.63 & 64 & 6.97 & 711.89 \\
\hline Severe reactions & 8 & 18.60 & 8 & 4.00 & 32 & 25.40 & 24 & 2.61 & $1 \quad 5.59$ \\
\hline No food allergy & 22 & 51.16 & 142 & 71.00 & 68 & 53.97 & 7830 & 90.41 & 182.52 \\
\hline Total & 43 & 100.0 & 200 & 100.0 & 126 & 100.0 & 918 & 100.0 & 100.0 \\
\hline
\end{tabular}

Anaphylaxis, urticaria, angio-oedema, bronchospasm.

PROF, profilins; LTP, lipid transfer protein; OAS, oral allergy syndrome.

sitized. In fact, in the northwestern Atlantic coast of Spain, $80 \%$ of the allergic population is sensitized only to grasses. In contrast, in dry semi-desert areas and on the Mediterranean coast, where pollen counts are lower, allergic patients are mainly polysensitized, with high s/gE titres, and react to pollens of different species that usually do not sensitize the population of grass pollen-dominated areas. In these regions, patients progress in sensitization complexity as they are sensitized to both major allergens and pan-allergens, as exemplified by the finding that simultaneous sensitization to profilin and polcalcin is linked to a twofold increase in sensitization to major allergens and a higher disease evolution time. As the preventive effect of specific immunotherapy in the development of new sensitizations has been reported $[27,28]$, it seems plausible that a high exposure to a single pollen species might play a similar role in the prevention of further sensitization. In fact, the overall prevalence of pollinosis is similar in all studied territories [29], regardless of pollen levels. One could argue that specific immunotherapy in monosensitized patients is helping an ongoing tolerance mechanism due to an added allergen load. In contrast, in the areas where patients are polysensitized, intervention strategies are not clear. The best therapeutic intervention for these patients, who present allergy symptoms most of the year, remains to be 


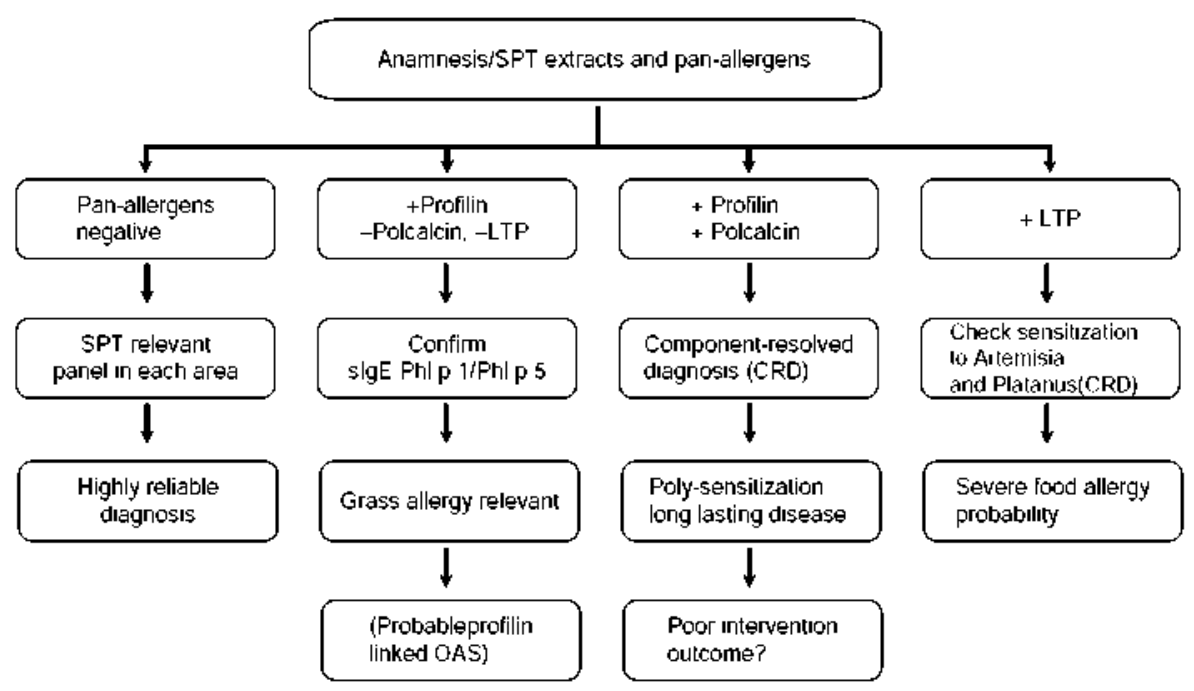

Fig. 4. Diagnosis algorithm combining skin prick tests (SPTs) to conventional extracts and pan-allergens, and a component-resolved diagnosis of pollen-allergic patients.

determined. The component-resolved diagnostic strategy leads to a more accurate determination of the sensitization profile of these patients, and consequently, to better intervention strategies.

To overcome the problems of in vivo testing of recombinant molecules, where complex regulatory frameworks make the development of new diagnostics expensive and unfeasible, we used a strategy with purified proteins from natural sources or fractions enriched with a specific allergen for SPT, in order to identify panallergen-sensitized patients. These new diagnostic extracts can be used to identify patients in whom conventional diagnostic procedures have a very limited value. On the other hand, pan-allergen-negative patients can be diagnosed using conventional procedures with a good diagnostic value and an optimum pharmacoeconomic balance. With this simple approach, the number of patients who have to be diagnosed by alternative molecular screening methods can be significantly reduced. In a rough evaluation, and depending on the area, $25 \%$ to more than $50 \%$ of patients had a different diagnosis when the new algorithm shown in Fig. 4 was applied, in comparison with the traditional diagnostic procedure. These data must have a clear incidence on immunotherapy formulae and could presumably influence the therapeutic outcome.

The link between pollen sensitization and food allergy was also studied. Profilin has been confirmed to behave as a minor grass allergen. Only some profilin-sensitized patients developed food allergy and presented mild symptoms. This could be because food allergy is more linked to an overall inflammation level and profilin might simply be a marker, as it is the only grass allergen present in foods. This is supported by the fact that there are high percentages of profilin-linked food symptoms in areas of extreme grass exposure, as occurs in some regions of Spain [1], as compared with intermediate exposure areas in North and Central Europe.

The high prevalence of sensitization to LTPS is noteworthy. The prevalence of LTP sensitization was lower in those areas where birch pollinosis was relevant. This finding had already been obtained [30], but there is no explanation for this inverse association. In contrast, there are areas where a $40 \%$ prevalence of sensitization to LTPS is detected. These areas have a low pollen allergen load and extensive orchard tree crops. In fact, $10 \%$ of the population of these areas, selected on the basis of having seasonal respiratory symptoms, was monosensitized to Pru p 3, which would support a respiratory sensitization route for LTPs, as has been previously suggested [31]. To rule out a possible sensitization to Platanus as a cause for Pru p 3 recognition, specific IgE to major Platanus allergens was performed. The prevalence was low and could not explain the high prevalence to Pru p 3, thus confirming the respiratory relevance of Pru p 3 sensitization.

The new diagnostic strategy defined here, where a combination of new pan-allergen SPTs and a component-resolved diagnosis is used, offers a significant improvement in diagnostic strategies that can be applied in daily practice. As an example, Table 5 shows three individual cases where the apparently polysensitized patients were found to have a more simple sensitization profile. Moreover, this new diagnostic approach will enable us to improve the efficacy of specific immunotherapy, avoiding the use of extract mixtures and concentrating the therapeutic action on the allergens that are actually responsible for the allergic symptoms. 
Table 5. Example of three individual cases where the new diagnosis allows a clear improvement in the determination of relevant sensitizations

\begin{tabular}{|c|c|c|c|}
\hline & Case 1 & Case 2 & Case 3 \\
\hline SPT Artemisia $\left(\mathrm{mm}^{2}\right)$ & 4 & 15 & 0 \\
\hline $\operatorname{slg} E(k U / L)$ Art v 1 & 0.06 & 0.27 & 0.12 \\
\hline \multicolumn{4}{|l|}{ SPT Betula $\left(\mathrm{mm}^{2}\right)$} \\
\hline $\operatorname{slgE}(\mathrm{kU} / \mathrm{L})$ Bet $v 1$ & 0.00 & 0.01 & 0.06 \\
\hline SPT Cupressus (mm²) & 12 & 16 & 0 \\
\hline slgE (kU/L) Cup s 1 & 0.01 & 0.04 & 0.00 \\
\hline $\begin{array}{l}\text { SPT Olea }\left(\mathrm{mm}^{2}\right) \\
\text { slgE }(\mathrm{kU} / \mathrm{L})\end{array}$ & 20 & 135 & 24 \\
\hline ole e 9 & 0.00 & 0.00 & 0.00 \\
\hline Ole e 1 & 0.02 & 1.84 & 0.02 \\
\hline SPT Parietaria $\left(\mathrm{mm}^{2}\right)$ & 6 & 49 & 0 \\
\hline slgE (kU/L)Par j 1 & 0.00 & 0.00 & 0.24 \\
\hline $\begin{array}{l}\text { SPT grass mix }\left(\mathrm{mm}^{2}\right) \\
\text { slgE (kU/L) }\end{array}$ & 99 & 54 & 25 \\
\hline Phl p 1 & 89.45 & 0.00 & 2.44 \\
\hline Phl p 5 & 67.80 & 10.88 & 1.26 \\
\hline SPT Platanus $\left(\mathrm{mm}^{2}\right)$ & 30 & 48 & 28 \\
\hline SPT Plantago $\left(\mathrm{mm}^{2}\right)$ & 25 & 42 & 54 \\
\hline slgE (kU/L) Pla I 1 & 0.00 & 0.07 & 32.55 \\
\hline SPT Polcalcin $\left(\mathrm{mm}^{2}\right)$ & 0 & 66 & 0 \\
\hline slgE (kU/L) Che a 3 & 0.00 & 3.48 & 0.00 \\
\hline SPT Profilin $\left(\mathrm{mm}^{2}\right)$ & 81 & 0 & 72 \\
\hline slgE (kU/L) Mal d 4 & 1.99 & 0.02 & 0.59 \\
\hline SPT Peach (mm²) & 4 & 96 & 0 \\
\hline slgE (kU/L) Ргu p 3 & 0.00 & 1.66 & 0.01 \\
\hline SPT Salsola $\left(\mathrm{mm}^{2}\right)$ & 12 & 80 & 6 \\
\hline slgE (kU/L) Sal k 1 & 0.01 & 0.03 & 0.02 \\
\hline
\end{tabular}

The first case is a patient monosensitive to grasses, the second to olive, grasses and peach LTP and the third mainly to the English plantain. SPT, skin prick test; slgE, specific lgE.

\section{Acknowledgements}

This study was supported by ALK-Abello S.A.

We would like to thank the EXPO2 study group for their excellent work and the participating investigators from the following Hospitals and private allergy practices: Asturias: Dr Gaspar Gala, Hospital Monte Naranco, Dr José A. Alvarez, Hospital Universitario Central de Asturias. Aragón: Zaragoza (Hospital Clínico, Hospital Royo Villanova, Clínica Lagasca), Huesca (Dr José Antonio Compaired), Teruel (Hospital Obispo Polanco). Baleares: Dr Albert Oehling, Dra Raquel Kraemer, Hospital Son Dureta, Hospital de Ibiza. Canarias: Tenerife (Hospital Virgen de la Candelaria). Cantabria: Hospital Marqués de Valdecilla, Alergocantabria, Hospital de Cantabria. Castilla-La Mancha: Guadalajara (Hospital General), Cuenca (Hospital Virgen de la Luz). Castilla-León: Burgos (Hospital Gral. Yagüe), Soria (Hospital General), Zamora (Hospital Virgen de la Concha), Salamanca (Hospital General, Clínica Alergoasma, Dr Javier Muñoz), Segovia (Hospital General (Adultos e Infantil), Ávila (Hospital Ntra. Sra. de Sonsoles (Adultos e Infantil), Valladolid (Hospital Rio Hortega, Gabinete Médico Paracelso), Palencia (Hospital Rio Carrión), León (Hospital San Juan de Dios, Hospital Comarcal de El Bierzo). Cataluna: Girona (Hospital Josep Trueta), Barcelona (Hospital Bellvitge, Dr Marcel Ibero), Lleida (Hospital Santa María), Extremadura: Cáceres (Hospital Virgen del Puerto). Galicia: A Coruna (Hospital Juan Canalejo, Hospital de Conxo, Hospital Clínico Santiago, Hospital Arquitecto Marcide), Lugo (Hospital Xeral Calde, Hospital Burela), Ourense (Hospital Santa $M^{a}$ Nai), Pontevedra (Hospital Provincial, Hospital Xeral Vigo). La Rioja: Hospital San Millán y San Pedro, Fundación Hospital de Calahorra. Navarra: Hospital Virgen del Camino, Clínica Universitaria de Navarra. Pais Vasco: Vizcaya (Hospital de Basurto, Hospital de Galdacano, Hospital de Cruces (Adultos e Infantil), Guipúzcoa (Hospital de Donostia, Hospital de Mendaro, Hospital de Zumárraga), Álava (Hospital Santiago Apóstol).

We would like to thank Ms Carmen Barrio for her expert secretarial support.

\section{References}

1 Barber D, De la Torre F, Feo F et al. Understanding patient sensitization profiles in complex pollen areas. A molecular epidemiological study. Allergy 2008; 63:550-1558.

2 Asero R, Monsalve R, Barber D. Profilin sensitization detected in the office by skin prick test: a study of prevalence and clinical relevance of profilin as a plant allergen. Clin Exp Allergy 2008; 38:1033-7.

3 King TP, Hoffman F, Lowenstein H, Marsh DG, Platts-Mills T, Thomas W. Allergen nomenclature. IUIS/WHO Allergen Nomenclature Subcommittee. Bull World Health Organ 1994; 72: $797-806$.

4 Stumvoll S, Westritschnig K, Lidholm J et al. Identification of cross-reactive and genuine Parietaria judaica pollen allergens. J Allergy Clin Immunol 2003; 111:974-9.

5 Andersson K, Lidholm J. Characteristics and immunobiology of grass pollen allergens. Int Arch Allergy Immunol 2003; 130: 87-107.

6 Pittner G, Vrtala S, Thomas WR et al. Component-resolved diagnosis of house-dust mite allergy with purified natural and recombinant mite allergens. Clin Exp Allergy 2004; 34:597-603.

7 Radauer $\mathrm{C}$, Breiteneder $\mathrm{H}$. Pollen allergens are restricted to few protein families and show distinct patterns of species distribution. J Allergy Clin Immunol 2006; 117:141-7.

8 Chapman MD, Pomés A, Breiteneder H, Ferreira F. Nomenclature and structural biology of allergens. J Allergy Clin Immunol 2007; 119:414-20.

9 Ledesma A, Barderas $R$, Westritschnig $K$ et al. A comparative analysis of the cross-reactivity in the polcalcin family including Syr $\vee 3$, a new member from lilac pollen. Allergy 2006; 61: $477-84$.

10 Barderas R, Villalba M, Rodriguez R. Recombinant expression, purification and cross-reactivity of chenopod profilin: rChe a 2 as a good marker for profilin sensitization. Biol Chem 2004; $385: 731-7$. 